\title{
Fabrication of Ni-W Microgears Using LIGA Process
}

\author{
Kohzo Tamada, ${ }^{1 *}$ Sho Amano, ${ }^{1}$ Kana Okamoto, ${ }^{2}$ Masaya Takeuchi,,${ }^{1,3}$ \\ Akinobu Yamaguchi, ${ }^{1}$ Yuichi Utsumi, ${ }^{1}$ and Tohru Yamasaki ${ }^{2}$ \\ ${ }^{1}$ Laboratory of Advanced Science and Technology for Industry, University of Hyogo, \\ 3-1-2 Kouto, Kamigori, Ako-gun, Hyogo 678-1205, Japan \\ ${ }^{2}$ Research Center for Advanced Metallic Materials, University of Hyogo, \\ 2167 Shosha, Himeji, Hyogo 671-2280, Japan \\ ${ }^{3}$ Graduate School of Engineering, University of Hyogo, 2167 Shosha, Himeji, Hyogo 671-2280, Japan
}

(Received October 26, 2021; accepted December 13, 2021)

Keywords: microgear, Ni-W electrodeposited alloys, LIGA process, deep X-ray lithography, microfabrication

Microgears made of $\mathrm{Ni}-\mathrm{W}$ alloy that are expected to have high strength and ductility, i.e., high durability, were successfully fabricated by using the LIGA process with synchrotron radiation. Ni-W electrodeposited alloys have a Vickers hardness of about HV 600 and a tensile strength of about $2000 \mathrm{MPa}$ or above for a sample thickness of up to about $20 \mu \mathrm{m}$. However, they lose their excellent mechanical properties with increasing sample thickness. For this reason, $\mathrm{Ni}-\mathrm{W}$ electrodeposited alloys have not yet been used in the LIGA process for the fabrication of microcomponents. We consider that the decrease in strength is due to the increased electric resistance of the $\mathrm{Ni}-\mathrm{W}$ film during electrodeposition. To solve this problem, we developed a new process in which a copper reversing mold is fabricated in the first step, then $\mathrm{Ni}-\mathrm{W}$ alloy is electrodeposited on the copper reversing mold to prevent the electric resistance from increasing in the second step as the mold forming process. Using this process, microgears with reference diameters from 1000 to $80 \mu \mathrm{m}$ were fabricated with high dimensional accuracy.

\section{Introduction}

Microturbines are an important component of microfluidic systems. Microgears have similar shapes to microturbines and are required to meet the increasing demand for ultrasmall motors in various fields, especially the medical field. Both microturbines and microgears are required to have high dimensional accuracy and durability, but it is difficult to achieve high dimensional accuracy on the order of microns for metals in conventional machining. For example, the minimum reference diameter of gears in practical use is about $300 \mu \mathrm{m}$, and it is difficult to fabricate high-precision gears with a reference diameter of less than $300 \mu \mathrm{m} .{ }^{(1)}$

On the other hand, the LIGA (an acronym for Lithographie, Galvanoformung und Abformung in German) process $(2$, Note 1) using synchrotron radiation can be used to fabricate metallic components with high aspect ratio and submicron-level dimensional accuracy. However, in the electroplating step of the LIGA process, only soft metals such as $\mathrm{Ni}$ and $\mathrm{Cu}$ can be deposited,

*Corresponding author: e-mail: ej21i013@steng.u-hyogo.ac.jp

https://doi.org/10.18494/SAM.2021.3712 
and therefore it has been difficult to fabricate metallic microparts with high hardness and durability. For example, Ni-Co electrodeposited alloy is a hard material with a Vickers hardness of HV 400, but a long time is generally required to deposit a thick layer of hard alloy plating owing to the low electrodeposition speed, and the layer may peel off from the substrate owing to residual stress.

In this study, the Ni-W electrodeposited alloy ${ }^{(3-14)}$ developed by Yamasaki and others, which showed high strength and ductility, has been applied to the LIGA process using synchrotron radiation to fabricate high-precision microgears with high durability. Ni-W electrodeposited alloys have nanocrystalline/amorphous composite structures with high plastic deformability despite their high strength. However, electrodeposited films of these alloys exhibit severe brittleness with increasing thickness. To solve this problem, we have developed a new process of double plating using a copper reversing mold. As a result, we succeeded in fabricating microgears made of $\mathrm{Ni}-\mathrm{W}$ electrodeposited alloy with high plastic durability, and we evaluated their shape accuracy.

Note 1: The LIGA process was developed at Karlsruhe Nuclear Research Center (now Karlsruhe Institute of Technology) in Germany in the 1970s to fabricate micro-metallic mechanical parts, which are the components of micromechanisms. The LIGA process consists of three steps: lithography (Lithographie), electroplating (Galvanoformung), and molding (Abformung). After the fabrication of a polymer (resist) microstructure by lithography, a mold is fabricated by electroplating. In the molding process, various materials such as resins, metals, and ceramics are processed to replicate the microstructures of the mold. This technology attracted worldwide attention in the $1990 \mathrm{~s}$ as a completely new microfabrication technique. However, this technique is not yet in widespread use owing to the lack of electrodeposited alloys with sufficient strength to be used as metal microparts.

\section{Materials and Methods}

\subsection{Double electroplating in LIGA process}

In this study, the Ni-W alloy having about 17 at.\% W with a Vickers hardness of about HV 600 and a tensile strength of about $2500 \mathrm{MPa}$ was used as the electrodeposited alloy in the LIGA process. When the Ni-W alloy was directly deposited on an electrically insulating resist microstructure fabricated by lithography, the deposited alloy retained its high strength and ductility up to a thickness of about $20 \mu \mathrm{m}$. However, when the thickness exceeded $20 \mu \mathrm{m}$, the strength decreased owing to embrittlement. This may be due to the increase in electric resistance during electrodeposition, i.e., the electric current flowed only from the $\mathrm{Cu}$ substrate (resistance of $\left.\mathrm{Cu} \sim 1.68 \times 10^{-8} \Omega \cdot \mathrm{m}\right)$ and the electric resistance increased with the thickness of the electrodeposited Ni-W alloy (resistance of Ni-W $\sim 7 \times 10^{-7} \Omega \cdot \mathrm{m}$ ).

Thus, we developed a new process. As shown in Fig. 1, a metal reversing mold was made by electroforming copper sulfate in the first electroforming process, and then $\mathrm{Ni}-\mathrm{W}$ electroforming was performed as the second electroforming process in mold forming. In this process, because the current can flow not only through the substrate but also through the copper reversing mold, the increase in resistance can be prevented. We expected that high-strength Ni-W layers would be formed on the substrate and sidewall surfaces of a structure in the initial stage of electrodeposition, enabling the fabrication of high-strength microgears. 


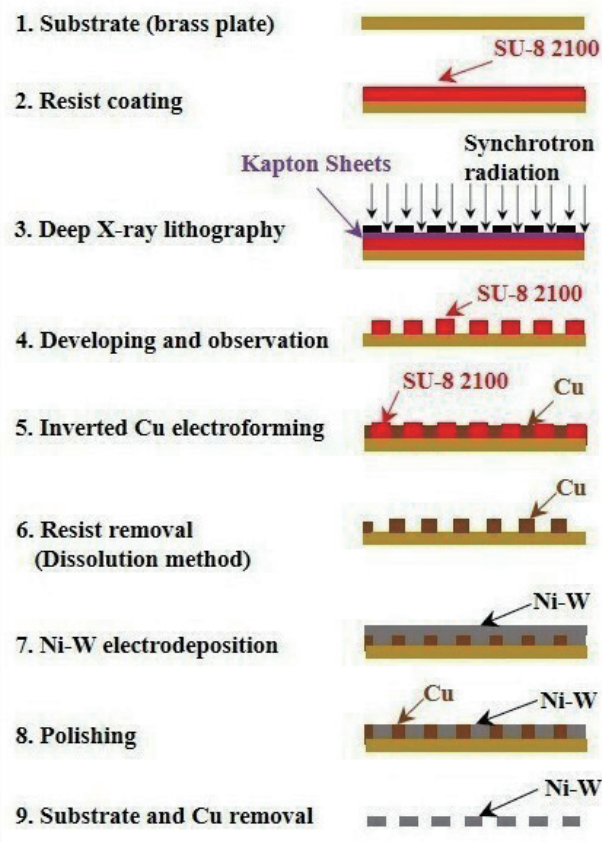

Fig. 1. (Color) Flowchart of production process of microgears.

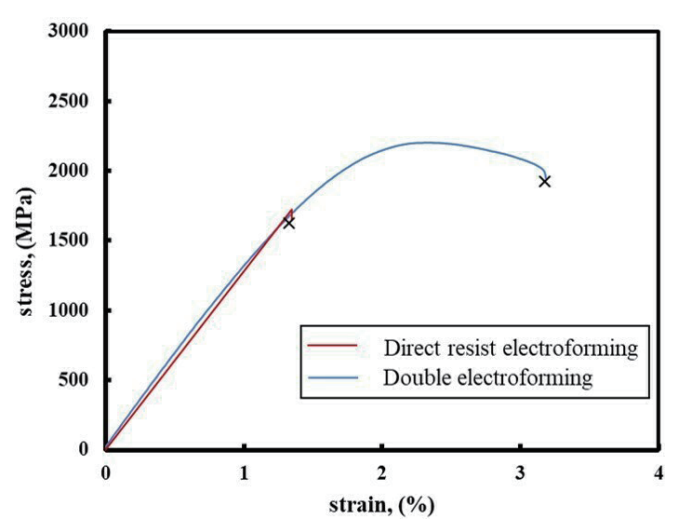

Fig. 2. (Color) Stress-strain curves obtained by tensile testing of micro-tensile specimens fabricated by direct resist electroforming and double electroforming.

Figure 2 shows the results of tensile tests of micro-tensile specimens with a thickness of 60 $\mu \mathrm{m}$ produced by double electroforming, compared with those of specimens obtained by conventional direct electroforming. As shown in Fig. 2, the nominal tensile strength of the directly deposited Ni-19 at.\% W alloy is about $1600 \mathrm{MPa}$, while that of the alloy fabricated by the new process shown in Fig. 1 is about $2200 \mathrm{MPa}$. The ductility of the alloy fabricated by double electroforming was higher than that of the alloy fabricated by conventional direct electroforming, and its strain was about twice as high. From these results, it was confirmed that the deposition of $\mathrm{Ni}-\mathrm{W}$ on a copper reversing mold is effective for obtaining a film of about $20 \mu \mathrm{m}$ thickness without any deterioration of its strength and ductility. ${ }^{(8)}$

\subsection{Deep X-ray lithography}

X-ray exposure for resist processing was performed at the LIGA beamline (BL11) of the New SUBARU synchrotron radiation facility of the University of Hyogo. The electron storage energy of New SUBARU is $1 \mathrm{GeV}$, the storage current is $300 \mathrm{~mA}$, and X-rays of 2 to $6 \mathrm{keV}$ are supplied. ${ }^{(15)}$

The X-ray mask was a self-made membrane mask with $\mathrm{Au}$ as a light-shielding material. Resist processing was attempted with various gear patterns. Microgear inversion patterns were fabricated with four different numbers of gear teeth $(12,14,17$, and 24), twelve different reference diameters $\left(80\right.$ to $1000 \mu \mathrm{m}$ ), and two pressure angles $\left(20\right.$ and $\left.25^{\circ}\right)$. The addendum modification coefficient was 0, 0.05, 0.1, or 0.35. SU-8 2100 (Nippon Kayaku Co., Ltd.) was used 
as the resist and applied to a brass substrate (YAMAMOTO-MS Co., Ltd.) with a thickness of about $100 \mu \mathrm{m}$ using a spin coater. After the coating, the resist was preheated using a hot plate at $60{ }^{\circ} \mathrm{C}$ for $5 \mathrm{~min}$, then at $75^{\circ} \mathrm{C}$ for $2 \mathrm{~h}$. A Kapton film (Du Pont-Toray Co., Ltd.) was sandwiched between the X-ray mask and the resist, and contact lithography was carried out. The resist sample was scanned at a speed of $v=5 \mathrm{~mm} / \mathrm{s}$ during X-ray exposure to standardize the exposure intensity. The X-ray dose was set to $15 \mathrm{~J} / \mathrm{cm}^{2}$.

After the exposure, the resist was postheated at $60^{\circ} \mathrm{C}$ for $5 \mathrm{~min}$ and at $75^{\circ} \mathrm{C}$ for $2 \mathrm{~h}$. Next, the resist was immersed in SU-8 developer (Nippon Kayaku) to induce its reaction and produce the structure. Since SU-8 is a negative photoresist, the exposed area does not react and remains unchanged.

\subsection{First electroforming process}

Copper sulfate was electrodeposited on the resist microgear structure formed by X-ray lithography as a primary electroforming process. A copper reversing mold with a thickness of about $60 \mu \mathrm{m}$ was fabricated in this electrodeposition. To prevent its surface oxidation, the copper reversing mold was immersed in a discoloration inhibitor (Top Rinse CU-5, Okuno Chemical Industries Co., Ltd.) for $2 \mathrm{~min}$, washed with water, and then quickly dried with a dryer. After that, the resist remaining on the mold was dissolved and removed using a remover. First, the substrate was immersed in Remover PG (Nippon Kayaku) at $70^{\circ} \mathrm{C}$ for $2 \mathrm{~h}$ to swell the resist and then washed with water. Next, the resist was stripped in a 1:1 mixture of Remover K (Parts A and B) (Nippon Kayaku) at $70{ }^{\circ} \mathrm{C}$ for $1 \mathrm{~h}$ and rinsed twice. Finally, it was neutralized with Neutralizer K (Nippon Kayaku) and rinsed with water and dried with ethanol.

\subsection{Second electroforming (replication) process}

Microgears were fabricated by $\mathrm{Ni}-\mathrm{W}$ electrodeposition on a copper reversing mold as a secondary electroforming process. The plating solution was a mixture of nickel sulfate and sodium tungstate, with citric acid and ammonium sulfate added as complexing agents. An Ir-Ta mesh was used as an anode plate on the resist-removed brass substrate. ${ }^{(4,7)}$ In addition, the brushing technique proposed by Nakayama and coworkers. was applied, which can suppress the generation of voids and pits due to hydrogen bubbles. ${ }^{(4,8)}$ The plating time was adjusted so that the film thickness was approximately $100 \mu \mathrm{m}$. The electrodeposited Ni-W microgears were immersed in $\mathrm{HNO}_{3}$ solution to separate them from the brass substrate and the copper reversing mold. ${ }^{(4)}$

\section{Reproducibility}

Scanning electron microscopy (SEM; VE-7800, Keyence Corporation) was used to observe the fabricated Ni-W alloy microgears. The acceleration voltage was $10 \mathrm{kV}$. Figure 3 shows SEM images of microgears having diameters of $1000,500,300$, and $80 \mu \mathrm{m}$. These images show that 


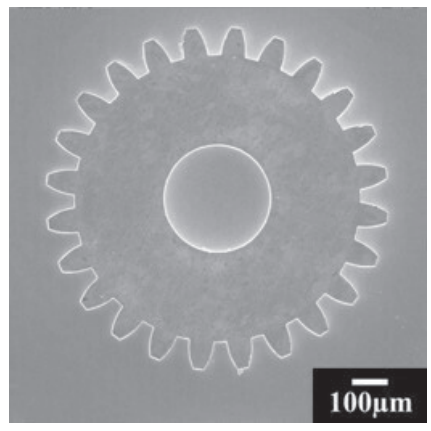

(a)

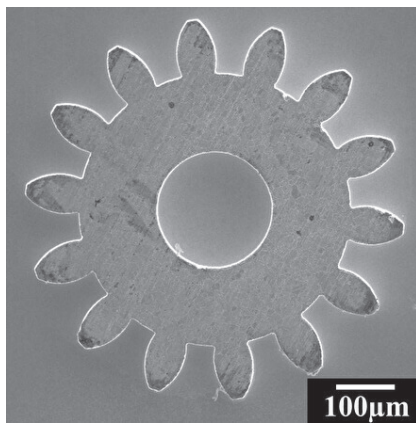

(b)

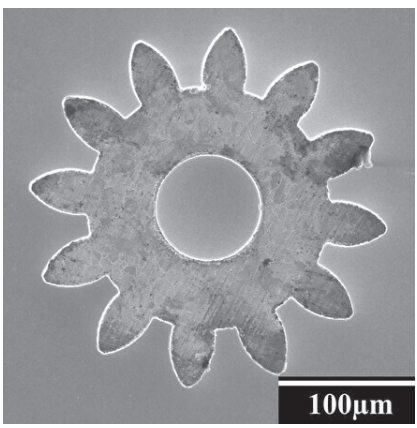

(c)

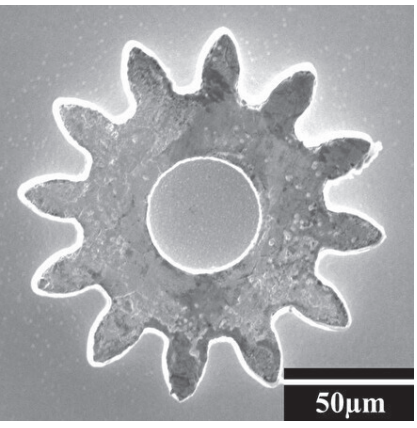

(d)

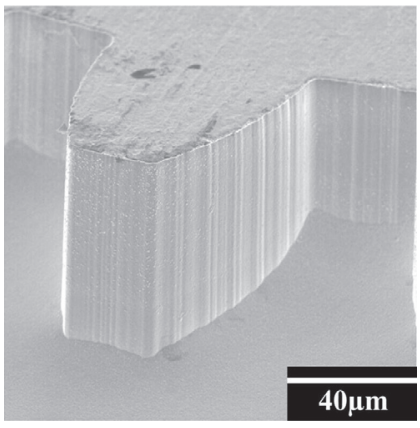

(e)

Fig. 3. SEM images of Ni-W microgears: (a) $\varphi 1000 \mu \mathrm{m}$, (b) $\varphi 500 \mu \mathrm{m}$, (c) $\varphi 300 \mu \mathrm{m}$, (d) $\varphi 80 \mu \mathrm{m}$, and (e) enlargement of part of (b).

Table 1

Shape evaluation of Ni-W microgears: (a) $\varphi 1000 \mu \mathrm{m}$, (b) $\varphi 500 \mu \mathrm{m}$, (c) $\varphi 300 \mu \mathrm{m}$, and (d) $\varphi 80 \mu \mathrm{m}$.

\begin{tabular}{lcccccccc}
\hline & \multicolumn{2}{c}{$(\mathrm{a})$} & \multicolumn{2}{c}{$(\mathrm{b})$} & \multicolumn{2}{c}{$(\mathrm{c})$} \\
& $\begin{array}{c}\text { CAD } \\
\text { dimensions } \\
(\mu \mathrm{m})\end{array}$ & $\begin{array}{c}\text { Average } \\
\text { value } \\
(\mu \mathrm{m})\end{array}$ & $\begin{array}{c}\text { CAD } \\
\text { dimensions } \\
(\mu \mathrm{m})\end{array}$ & $\begin{array}{c}\text { Average } \\
\text { value } \\
(\mu \mathrm{m})\end{array}$ & $\begin{array}{c}\text { CAD } \\
\text { dimensions } \\
(\mu \mathrm{m})\end{array}$ & $\begin{array}{c}\text { Average } \\
\text { value } \\
(\mu \mathrm{m})\end{array}$ & $\begin{array}{c}\text { CAD } \\
\text { dimensions } \\
(\mu \mathrm{m})\end{array}$ & $\begin{array}{c}\text { Average } \\
\text { value } \\
(\mu \mathrm{m})\end{array}$ \\
\hline Tip diameter & 1075 & 1041 & 583 & 556 & 350 & 340 & 94 & 87 \\
Reference diameter & 1000 & 966 & 500 & 479 & 300 & 291 & 80 & 75 \\
Inside diameter & 333 & 322 & 167 & 164 & 100 & 98 & 26 & 24 \\
\hline Shape error $(\%)$ & \multicolumn{6}{c}{3.3} & \multicolumn{3}{c}{3.5} & & 2.6 & & 7.1
\end{tabular}

microgears with a fine metallic luster could be fabricated in accordance with the designed mask pattern. Their thicknesses were measured to be about $60 \mu \mathrm{m}$.

From the SEM images in Figs. 3(a)-3(d), the tooth tip, reference circle, and inner circle diameters were measured at three different locations, and their averages were calculated and compared with the CAD dimensions to obtain the shape error. The results are shown in Table 1. From the results, we conclude that microgears can be fabricated with accuracies of several percent by this process. 


\section{Summary and Conclusions}

We succeeded in fabricating microgears made of Ni-W electrodeposited alloy using the LIGA process. We developed a new double plating method that involved the production of a copper reversing mold. The Ni-W electrodeposited alloy is expected to enable the production of highly durable precision microgears with a Vickers hardness of about HV 600 and a tensile breaking strength of about $2200 \mathrm{MPa}$ because of its high strength and ductility. The results of this study are summarized below.

(1) Microgears with a maximum reference diameter of $1000 \mu \mathrm{m}$ and a minimum diameter of 80 $\mu \mathrm{m}$ were fabricated.

(2) As in Table 1, the shape error between the design and the fabricated microgears was evaluated to be several percent.

We are planning to clarify the material properties (composition, crystallite size, and hardness) of the gears and conduct dynamic property tests (wear and endurance tests) on interlocked gears to demonstrate their use as highly durable microgears. Using the same technology, a highly durable microturbine can be realized, which will contribute to further research on microfluidics.

\section{References}

1 Y. Kitakoga: Int. J. Jpn. Soc. Precis. Eng. 58 (1992) 1284 (in Japanese). https://doi.org/10.2493/jjspe.58.1284

2 N. Nakajima and A. Umeda: Advanced Machining Technology \& Development Association: LIGA Process Advancing Three-Dimensional Ultrafine Machining Technology (Nikkan Kogyo Shimbun, 1998) pp. 89-97 (in Japanese).

3 T. Yamasaki, M. Yamada, H. Adachi, T. Nabeshima, and Y. Yokoyama: Microsyst. Technol. 20 (2014) 1941. https://doi.org/10.1007/s00542-013-2005-7

4 S. Nakayama, H. Adachi, T. Nabeshima, T. Miyazawa, and T. Yamasaki: Sci. Adv. Mater. 8 (2016) 2082. https://doi.org/10.1166/sam.2016.2825

5 T. Yamasaki, M. Sonobe, and H. Yokoyama: Mater. Sci. Forum 561-565 (2008) 1295. https://doi.org/10.4028/ www.scientific.net/MSF.561-565.1295

6 H. Hosokawa, T. Yamasaki, N. Sugamoto, M. Tomizawa, K. Shimojima1, and M. Mabuchi: Mater. Trans. 48 (2004) 1807. https://doi.org/10.2320/matertrans.45.1807

7 T. Yamasaki: Scr. Mater. 44 (2001) 1497. https://doi.org/10.1016/S1359-6462(01)00720-5

8 S. Nakayama, H. Adachi, and T. Yamasaki: J. Alloys Compd. 643 (2015) S22. https://doi.org/10.1016/j. jallcom.2014.12.209

9 K. Fujita, K. Nagaoka, T. Suidu, T. Nabeshima, and T. Yamasaki: J. Jpn. Inst. Met. Mater. 77 (2013) 192 (in Japanese). https://doi.org/10.2320/jinstmet.J2012067

10 K. Fujita, T. Suidu, and T. Yamasaki: J. Jpn. Inst. Metals 75-76 (2011) 348 (in Japanese). https://doi.org/10.2320/ jinstmet.75.348

11 H. Yokoyama, T. Yamasaki, T. Kikuchi, and T. Fukami: Mater. Sci. Forum 561-565 (2007) 1295. https://doi. org/10.4028/www.scientific.net/MSF.561-565.1295

12 N. Oda, H. Matsuoka, T. Yamasaki, and T. Fukamis: J. Metastable Nanocryst. Mater. 24-25 (2005) 411. https:// doi.org/10.4028/www.scientific.net/JMNM.24-25.411

13 T. Nasu, M. Sakurai, T. Kamiyama, T. Usuki, O. Uemura, K. Tokumitsu, and T. Yamasaki: Mater. Sci. Eng., A 375-377 (2004) 163. https://doi.org/10.1016/j.msea.2003.10.144

14 A. S. M. A. Haseeb: Microsyst. Technol. 14 (2008) 379. https://doi.org/10.1007/s00542-007-0447-5

15 M. Takeuchi, A. Yamaguchi, and Y. Utsumi: J. Synchrotron Radiat. 26 (2019) 528. https://doi.org/10.1107/ S1600577518017939 